\title{
A CENTRAL-DIFFERENCE SCHEME FOR A PURE STREAM FUNCTION FORMULATION OF INCOMPRESSIBLE VISCOUS FLOW*
}

\author{
RAZ KUPFERMAN ${ }^{\dagger}$
}

\begin{abstract}
We present a numerical scheme for incompressible viscous flow, formulated as an equation for the stream function. The pure stream function formulation obviates the difficulty associated with vorticity boundary conditions. The resulting biharmonic equation is discretized with a compact scheme and solved with an algebraic multigrid solver. The advection of vorticity is implemented with a high-resolution central scheme that remains stable and accurate in the presence of large gradients. The accuracy and robustness of the method are demonstrated for high Reynolds number flows in a lid-driven cavity.
\end{abstract}

Key words. incompressible Navier-Stokes equations, vorticity, stream function, biharmonic equation, algebraic multigrid, high-resolution schemes

AMS subject classifications. 65M06, 65M55, 76D05

PII. S1064827500373395

1. Introduction. The vorticity formulation of the Navier-Stokes equations is a classical starting point for approximation methods, due to the distinguished role of vorticity in high Reynolds number flows. The difficulty with a vorticity formulation is the lack of natural boundary conditions; the no-slip boundary conditions do not have a simple counterpart in terms of vorticity. In the context of computational methods, the problem of vorticity boundary conditions has a long history, dating back to the 30 's [22]; it has received much attention within the context of vortex methods [6], and there exists a substantial amount of recent work (see, e.g., Goodrich and Soh [11], Auteri and Quartapelle [3], Anderson and Reider [2], and E and Liu [8, 7]).

Recently, Ben-Artzi, Fishelov, and Trachtenberg have developed a method of vorticity/stream function dynamics [4]. This method uses explicitly the space of functions in which the dynamics take place. Specifically, the stream function dynamics take place in the Sobolev space $H_{0}^{2}$, whereas the vorticity field resides in the image of $H_{0}^{2}$ under the action of the Laplace operator. At the end of every time step a provisional solution is projected back onto the right functional space, in analogy with the projection onto the space of divergence-free velocity fields in the primitive-variable formalism [5]. As a result, no reference to vorticity boundary conditions is needed, and instead, natural boundary conditions are imposed (as an integral part of the dynamics space) on the stream function. The scheme we present in this paper belongs to this category. Like the scheme in [4], it evolves the stream function within the abovementioned functional space, but rather than using a "predictor-corrector" approach, it does it via implicit time stepping. In both cases, the computational complexity is dominated by the solution of a linear system of biharmonic type.

The advection of vorticity has been implemented using the Kurganov-Tadmor (KT) scheme [16], which was developed in the context of hyperbolic conservation laws; this scheme has been shown to remain accurate and robust in the presence of large

${ }^{*}$ Received by the editors June 7, 2000; accepted for publication (in revised form) December 4, 2000; published electronically May 10, 2001. This research was supported in part by the LBNL Directed Research and Development Program.

http://www.siam.org/journals/sisc/23-1/37339.html

${ }^{\dagger}$ Institute of Mathematics, The Hebrew University, Jerusalem 91904 Israel (raz@math.huji.ac.il). 
gradients; at the same time, it shares the relative simplicity of the central differencing framework. In addition, the KT scheme has a well-behaved semidiscrete limit, and as a result, time stepping is not tied to the spatial discretization (except for the standard stability requirements). The independence of the spatial and temporal discretizations adds a degree of modularity that may greatly simplify subsequent adaptations and improvements.

The biharmonic viscous term is discretized by means of a compact stencil (see [1]), which simplifies the treatment of boundary conditions. The fourth-order elliptic equation is then solved with an algebraic multigrid (AMG) solver [13]. This technique can be adapted with little modification to more complicated systems and geometries.

In section 2 we present the flow equations in vorticity-stream function formulation and describe the difficulty associated with boundary conditions. In section 3 we investigate a linear model equation, $u_{x x t}-u_{x x x x}=0$, inspired by the stream function formulation of the Navier-Stokes equations. This system is simple enough to be completely tractable but is still rich enough to capture the issue of boundary conditions. We prove the convergence of an implicit scheme that uses a compact stencil. The convergence is with respect to the $H^{2}$ norm, which is the appropriate norm for a variable analogous to the stream function. In section 4 we extend the scheme of section 3 to the Navier-Stokes equations in a two-dimensional bounded domain. In section 5 we present numerical results for a classical benchmark problem: flow in a two-dimensional lid-driven cavity. The method is found to be accurate and robust up to a regime of high Reynolds numbers, in which the flow becomes highly unstable and generates convoluted vorticity patterns. The scheme seems to be able to resolve vorticity patterns almost down to the scale of a single mesh size.

2. The vorticity-stream function formulation. We consider incompressible viscous flow in a two-dimensional domain. The motion of the fluid is governed by the Navier-Stokes equations,

$$
\begin{gathered}
\frac{\partial \mathbf{u}}{\partial t}+(\mathbf{u} \cdot \boldsymbol{\nabla}) \mathbf{u}=-\nabla p+\nu \Delta \mathbf{u}, \\
\boldsymbol{\nabla} \cdot \mathbf{u}=0,
\end{gathered}
$$

where $\mathbf{u}=\mathbf{u}(\mathbf{x}, t)=(u(\mathbf{x}, t), v(\mathbf{x}, t))$ is the Eulerian velocity field, $p=p(\mathbf{x}, t)$ is the pressure, and $\nu$ is the kinematic viscosity. In a bounded domain $\Omega$ enclosed by rigid walls, the impermeability of the walls and the no-slip condition imply

$$
\mathbf{u}(\mathbf{x}, t)=\mathbf{U}(\mathbf{x}, t), \quad \mathbf{x} \in \partial \Omega, \quad t>0,
$$

where $\mathbf{U}$ is the velocity of the wall.

In terms of the vorticity field $\omega=(\boldsymbol{\nabla} \times \mathbf{u})_{z}=\partial_{x} v-\partial_{y} u$, the flow equations read

$$
\frac{\partial \omega}{\partial t}+(\mathbf{u} \cdot \nabla) \omega=\nu \Delta \omega,
$$

where $\mathbf{u}$ is obtained from $\omega$ through the div-curl relations

$$
\begin{gathered}
\boldsymbol{\nabla} \cdot \mathbf{u}=0, \\
(\boldsymbol{\nabla} \times \mathbf{u}) \cdot \hat{z}=\omega .
\end{gathered}
$$

The divergence condition implies that the flow field is derivable from a scalar stream function, $\psi(\mathbf{x}, t)$,

$$
\mathbf{u}=\nabla^{\perp} \psi=\left(-\frac{\partial \psi}{\partial y}, \frac{\partial \psi}{\partial x}\right)
$$


which, substituted into the curl condition, yields the Poisson equation

$$
(\boldsymbol{\nabla} \times \mathbf{u}) \cdot \hat{z}=\Delta \psi=\omega .
$$

Finally, the boundary conditions (2.2) translate into boundary conditions for the stream function

$$
\nabla^{\perp} \psi=\mathbf{U}, \quad \mathbf{x} \in \partial \Omega .
$$

The set of equations (2.3), (2.5), and (2.6), together with the boundary conditions, (2.7), is known as the vorticity-stream function formulation of the Navier-Stokes equations.

The classical difficulty with the vorticity-stream function formulation is the improper partition of boundary conditions. The presence of a dissipative term in (2.3) requires the specification of boundary conditions for the vorticity, but these are not prescribed explicitly. Vorticity boundary conditions are extremely important from a physical point of view as they represent the mechanism of vorticity generation at the boundary. On the other hand, the Poisson equation (2.6) is overdetermined by both Neumann and Dirichlet boundary conditions (2.7).

This difficulty is immediately removed if the vorticity equation (2.3) is interpreted instead as an equation for the stream function

$$
\frac{\partial}{\partial t} \Delta \psi+\left[\left(\nabla^{\perp} \psi\right) \cdot \nabla\right] \Delta \psi=\nu \Delta^{2} \psi .
$$

This equation contains a biharmonic operator so that the boundary conditions (2.7) are the natural ones with no over- or underdetermination.

3. A linear model equation. The issue of vorticity boundary conditions can be illustrated by considering a simple model equation inspired by (2.8): a fourth-order linear equation for a one-dimensional scalar field $u(x, t)$,

$$
\left\{\begin{array}{l}
u_{x x t}=u_{x x x x}, \quad x \in(0,1), \\
u(0, t)=u(1, t)=0, \\
u_{x}(0, t)=u_{x}(1, t)=0, \\
u(x, 0)=u_{0}(x),
\end{array}\right.
$$

where subscripts denote differentiation. Here $u$ plays a role analogous to the stream function, and $u_{x x}$ is the analogue of vorticity. We consider homogeneous boundary conditions; inhomogeneous ones are readily reduced to the homogeneous case by a standard change of variables [12].

Equation (3.1) is solvable by standard techniques, and its solution can be represented as a Fourier sine-series

$$
\begin{aligned}
u(x, t) & =\sum_{q=1}^{\infty} a_{q} \sin (\pi q x) e^{-\pi^{2} q^{2} t}+\int_{0}^{t} b_{1}(s) \sum_{q=1}^{\infty} \sin (\pi q x) e^{-\pi^{2} q^{2}(t-s)} d s \\
& +\int_{0}^{t} b_{2}(s) \sum_{q=1}^{\infty}(-1)^{q} \sin (\pi q x) e^{-\pi^{2} q^{2}(t-s)} d s,
\end{aligned}
$$

where

$$
a_{q}=\int_{0}^{1} u_{0}(x) \sin (\pi q x) d x
$$


and $b_{1}(t), b_{2}(t)$ are functions to be determined. The sine-series (3.2) automatically satisfies the Dirichlet boundary conditions $u(0, t)=u(1, t)=0$. The role of the functions $b_{1}(t), b_{2}(t)$ is to enforce the Neumann boundary conditions; they are determined implicitly by the conditions $u_{x}(0, t)=u_{x}(1, t)=0$. Note that

$$
\begin{array}{ll}
\lim _{s \rightarrow t} & \sum_{q=1}^{\infty} \sin (\pi q x) e^{-\pi^{2} q^{2}(t-s)}=\delta(x), \\
\lim _{s \rightarrow t} & \sum_{q=1}^{\infty}(-1)^{q} \sin (\pi q x) e^{-\pi^{2} q^{2}(t-s)}=\delta(1-x),
\end{array}
$$

which means that $b_{1}(t), b_{2}(t)$ can be regarded as the strength of point sources that are concentrated on the left and right boundary, respectively; they play a role analogous to vortex sheets in fluid mechanics.

The solution (3.2) can also be expanded in eigenfunctions,

$$
u(x, t)=\sum_{q} \alpha_{q} \varphi_{q}(x) e^{\Omega_{q} t},
$$

where the index $q$ runs over a discrete set of wavenumbers, and $\Omega_{q}=-\pi^{2} q^{2}$ is the corresponding amplification rate. The eigenfunctions, $\varphi_{q}$, divide into two families:

$$
\varphi_{q}^{(1)}(x)=[1-\cos (\pi q x)]
$$

where $q=2,4, \ldots$, and

$$
\varphi_{q}^{(2)}(x)=(2 / \pi q) \sin (\pi q x)-\cos (\pi q x)-2 x+1,
$$

where the wavenumbers $q$ are solutions of the transcendental equation

$$
\tan \frac{\pi q}{2}=\frac{\pi q}{2}
$$

Such wavenumbers are typical to a system with mixed boundary conditions [12]. The eigensolutions $\varphi_{q}^{(1)}$ correspond to the case where the boundary terms are identically zero: $b_{1}(t)=b_{2}(t) \equiv 0$ (no generation of "vortex sheets").

A natural approach in approximating (3.1) is to view it as an implicit equation for $u_{t}(x, t)$; this is analogous to the choice of stream function variables in fluid mechanics. For simplicity, it is sufficient to consider schemes that are first-order in time; the generalization to higher-order is straightforward. For example, a backward-Euler scheme reads

$$
\frac{u_{x x}^{n+1}-u_{x x}^{n}}{k}=u_{x x x x}^{n+1}
$$

where $k=t^{n+1}-t^{n}$ is the time step interval.

We discretize the unit segment using a regular mesh of $N+1$ points, with the first and last points coinciding with the left and right boundaries, $x_{0}=0, x_{N}=1$; the mesh spacing is $h=1 / N$. The standard discretizations of second and fourth derivatives involve stencils of three and five points, respectively, which implies that boundary conditions need to be prescribed at two points near each boundary. Alternatively, it 
is possible to use a compact 3-point stencil by introducing an auxiliary field $v$ that approximates $u_{x}$. Following [1], we propose the following scheme:

$$
\begin{aligned}
D^{0}\left(v_{j}^{n+1}-v_{j}^{n}\right) & =12 \lambda\left(D^{0} v_{j}^{n+1}-D^{+} D^{-} u_{j}^{n+1}\right), \\
D^{0} u_{j}^{n+1} & =\left(I+\frac{1}{6} h^{2} D^{+} D^{-}\right) v_{j}^{n+1},
\end{aligned}
$$

where $D^{0}, D^{ \pm}$are the standard central-, forward-, and backward-difference operators, $\lambda=k / h^{2}$, and $u_{0}=u_{N}=v_{0}=v_{N}=0$ at the boundary points. Equation (3.5) is a discrete differential-algebraic system; the first equation is an evolution equation, whereas the second is a constraint.

To prove that the numerical scheme (3.5) is convergent we first analyze its consistency and stability properties. Convergence follows from a generalization of Lax's theorem.

LEMma 3.1. The numerical scheme (3.5) is consistent with truncation error $\tau=O\left(h^{2}, k\right)$.

Proof. Let $u(x, t)$ be a smooth solution of $u_{x x t}=u_{x x x x}$, and let the discrete auxiliary field $v_{j}(t)$ be defined implicitly by

$$
D^{0} u\left(x_{j}, t\right)=\left(I+\frac{1}{6} h^{2} D^{+} D^{-}\right) v_{j}(t)
$$

with $v_{0}(t)=v_{N}(t)=0$. A Taylor expansion gives

$$
\left(I+\frac{1}{6} h^{2} D^{+} D^{-}\right)\left[u_{x}\left(x_{j}, t\right)-v_{j}(t)\right]=O\left(h^{4}\right),
$$

from which we conclude that

$$
v_{j}(t)=u_{x}\left(x_{j}, t\right)+O\left(h^{4}\right) .
$$

Substituting (3.6) into the first equation in (3.5) and performing another Taylor expansion, we finally obtain

$$
D^{0}\left[v_{j}(t+k)-v_{j}(t)\right]=12 \lambda\left[D^{0} v_{j}(t+k)-D^{+} D^{-} u\left(x_{j}, t+k\right)\right]+k \tau_{j}^{n}
$$

with $\tau_{j}^{n}=O\left(h^{2}, k\right)$.

LEMma 3.2. The numerical scheme (3.5) is unconditionally stable.

Proof. It is possible to construct two families of eigenvectors analogous to (3.3), (3.4) that span the space of solutions of (3.5). Specifically,

$$
u_{j}^{n}=\sum_{q} \alpha_{q} \tilde{\varphi}_{q}\left(x_{j}\right) \tilde{\Omega}_{q}^{n},
$$

where the eigenvectors that correspond to (3.3) are

$$
\tilde{\varphi}_{q}^{(1)}\left(x_{j}\right)=1-\cos \left(\pi q x_{j}\right)
$$

for $q=2,4, \ldots, N-1$, and the eigenvectors that correspond to (3.4) are

$$
\tilde{\varphi}_{q}^{(2)}\left(x_{j}\right)=\left(2 / A_{q}\right) \sin \left(\pi q x_{j}\right)-\cos \left(\pi q x_{j}\right)-2 x_{j}+1
$$


with

$$
\tan \frac{\pi q}{2}=\frac{A_{q}}{2}, \quad A_{q}=\frac{3 \sin (\pi q h)}{h[2+\cos (\pi q h)]} .
$$

The amplification factor $\tilde{\Omega}_{q}$ is given in both cases by

$$
\Omega_{q}=\left(1+\frac{4 k}{h^{2}} \tan ^{2} \frac{\pi q h}{2}\right)^{-1} .
$$

It is readily verified that (3.7) and (3.8) form a total of $n-1$ eigenvectors and thus span the space of solutions. For all values of $q$ the amplification factor $\left|\Omega_{q}\right|$ is strictly less than one, from which follows that all eigenmodes decay and the scheme is stable.

THEOREM 3.3. The numerical scheme (3.5) is convergent.

Proof. The proof is essentially a generalization of Lax's theorem. It relies on the facts that the truncation errors are small (consistency), and that there is no mechanism that amplifies errors (stability).

Let $u(x, t)$ be a smooth solution of $(3.1)$, let $u_{j}^{n}$ be a numerical solution of (3.5), and let $e_{j}^{n}=u(j h, n k)-u_{j}^{n}$ be the global error. By virtue of Lemma 3.1 and the linearity of (3.5), we obtain the equation for the error

$$
\begin{gathered}
D^{0}\left(w_{j}^{n+1}-w_{j}^{n}\right)=12 \lambda\left(D^{0} w_{j}^{n+1}-D^{+} D^{-} e_{j}^{n+1}\right)+k \tau_{j}^{n}, \\
D^{0} e_{j}^{n+1}=\left(I+\frac{1}{6} h^{2} D^{+} D^{-}\right) w_{j}^{n+1},
\end{gathered}
$$

where $w_{j}^{n}$ is the auxiliary field associated with $e_{j}^{n}$.

We next expand the error in the discrete eigenmodes (3.7), (3.8),

$$
e_{j}^{n}=\sum_{q} \alpha_{q}^{n} \tilde{\varphi}_{q}\left(x_{j}\right)
$$

Substitution of (3.10) into the auxiliary equation in (3.9) gives

$$
w_{j}^{n}=\sum_{q} \alpha_{q}^{n} \tilde{\psi}_{q}\left(x_{j}\right)
$$

where

$$
\begin{gathered}
\tilde{\psi}_{q}^{(1)}\left(x_{j}\right)=A_{q} \sin \left(\pi q x_{j}\right) \\
\tilde{\psi}_{q}^{(2)}\left(x_{j}\right)=2 \cos \left(\pi q x_{j}\right)+A_{q} \sin \left(\pi q x_{j}\right)-2
\end{gathered}
$$

are the two families of functions that correspond to $\tilde{\varphi}_{q}^{(1)}$ and $\tilde{\varphi}_{q}^{(2)}$. Noting that

$$
D^{0} \tilde{\psi}_{q}\left(x_{j}\right)=\frac{h A_{q}}{4} \frac{\sin (\pi q h)}{\sin ^{2}\left(\frac{1}{2} \pi q h\right)} D^{+} D^{-} \tilde{\varphi}_{q}\left(x_{j}\right)=\frac{3 \cos ^{2}\left(\frac{1}{2} \pi q h\right)}{1+2 \cos ^{2}\left(\frac{1}{2} \pi q h\right)} D^{+} D^{-} \tilde{\varphi}_{q}\left(x_{j}\right),
$$

we substitute (3.11) into the first equation in (3.9) and obtain after some basic manipulations

$$
\sum_{q}\left(\tilde{\Omega}_{q}^{-1} \alpha_{q}^{n+1}-\alpha_{q}^{n}\right) D^{0} \tilde{\psi}_{q}\left(x_{j}\right)=k \tau_{j}^{n}, \quad j=1,2, \ldots, N-1 .
$$


Now let $\chi_{q}\left(x_{j}\right)=D^{0} \tilde{\psi}_{q}\left(x_{j}\right)$; these functions span the space of grid functions defined over the $N-1$ inner points $x_{j}, j=1,2, \ldots, N-1$. Let $(f, g)=\sum_{j+1}^{N-1} h f_{j} g_{j}$ denote the discrete inner product, and let $B$ be the $(N-1) \times(N-1)$ matrix whose entries are $B_{q, q^{\prime}}=\left(\chi_{q}, \chi_{q^{\prime}}\right)$.

To obtain an explicit recursion relation for the $\alpha_{q}^{n}$, we take the scalar product of (3.12) with $\chi_{q^{\prime}}$ and invert by multiplying on the left by $B^{-1}$; thus

$$
\alpha_{q}^{n+1}=\tilde{\Omega}_{q} \alpha_{q}^{n}+k \tilde{\Omega}_{q} \sum_{q^{\prime}} B_{q, q^{\prime}}^{-1}\left(\chi_{q^{\prime}}, \tau^{n}\right),
$$

which by the discrete Duhammel principle gives

$$
\alpha_{q}^{n}=\tilde{\Omega}_{q}^{n} \alpha_{q}^{0}+k \sum_{r=0}^{n-1} \tilde{\Omega}_{q}^{n-r} \sum_{q^{\prime}} B_{q, q^{\prime}}^{-1}\left(\chi_{q^{\prime}}, \tau^{r}\right) .
$$

The first term of the right-hand side represents the amplification of the initial error, whereas the second represents the accumulation of the local truncation errors.

The vector which we are going to estimate is $D^{0} w^{n}=\sum_{q} \alpha_{q}^{n} \chi_{q}$, which is equivalent to the second derivative of the error $e^{n}$. If $\left\|D^{0} w^{n}\right\|_{2} \rightarrow 0$ as $h, k \rightarrow 0$, then the scheme converges in the $H^{2}$ norm, which is indeed the relevant norm for $u$ [4]. Using (3.13), the Cauchy-Schwarz inequality, and the fact that $0<\Omega_{q}<1$, we find

$$
\begin{aligned}
\left\|D^{0} w^{n}\right\|_{2}^{2} & =\sum_{q, q^{\prime}} \alpha_{q}^{n} B_{q, q^{\prime}} \alpha_{q^{\prime}}^{n} \\
& \leq\left\|D^{0} w^{0}\right\|_{2}^{2}+2 k n\left\|D^{0} w^{0}\right\|_{2}\|\tau\|_{2}+(k n)^{2}\|\tau\|_{2}^{2},
\end{aligned}
$$

where $\|\tau\|_{2}=\max _{n}\left\|\tau^{n}\right\|_{2}$. We need only the initial conditions to converge at least as $O\left(h^{2}\right)$ to conclude with the aid of Lemma 3.2 that the scheme converges in $H^{2}$, and that the order of convergence is $O\left(h^{2}, k\right)$.

4. The numerical scheme. Inspired by the model equation presented in the previous section, we construct an approximation scheme for (2.8). The temporal and the spatial discretizations are considered separately; this is legitimate when the scheme has a well-behaved semidiscrete limit [16].

4.1. Temporal discretization. Let $\psi^{n}$ denote the stream function at time $t^{n}$. We approximate (2.8) by a discretization that is second-order in time:

$$
\begin{aligned}
& \left(\Delta-\frac{1}{4} \nu k \Delta^{2}\right) \psi^{n+\frac{1}{2}}=\left(\Delta+\frac{1}{4} \nu k \Delta^{2}\right) \psi^{n}-\frac{1}{2} k[(\mathbf{u} \cdot \boldsymbol{\nabla}) \omega]^{n}, \\
& \left(\Delta-\frac{1}{2} \nu k \Delta^{2}\right) \psi^{n+1}=\left(\Delta+\frac{1}{2} \nu k \Delta^{2}\right) \psi^{n}-k[(\mathbf{u} \cdot \boldsymbol{\nabla}) \omega]^{n+\frac{1}{2}}
\end{aligned}
$$

that is, we use Crank-Nicholson for the viscous term and a midpoint rule for the advection term.

4.2. Spatial discretization. We discretize the system on a rectangular grid with fixed mesh spacing, $\Delta x=\Delta y=h$; a generalization to more complicated metrics will be presented elsewhere. The examples below are for a square domain, where the outermost grid points coincide with the boundaries of the system. We assume that at the beginning of each time step we possess second-order approximations for $\psi$ and its first derivatives - the two velocity components - at the grid points $\left(x_{i}, y_{j}\right)$, which we denote by $\psi_{i, j}, u_{i, j}$, and $v_{i, j}$, respectively. 
4.2.1. The advection term. We start with the advection term, which describes the conservative transport of vorticity along streamlines. Due to the incompressibility of the flow, it can be written in an equivalent conservative form,

$$
(\mathbf{u} \cdot \nabla) \omega=\nabla \cdot(\omega \mathbf{u})
$$

where the vector field $\omega \mathbf{u}$ is the vorticity flux.

The numerical analysis of nonlinear advection has been studied extensively in the context of hyperbolic systems of conservation laws (see, e.g., [10, 17]). Considerable effort has been devoted to the construction of so-called high-resolution schemes, which are designed to capture the structure of singularities, such as shocks and rarefaction waves. Although incompressible flows do not form shocks, experience shows that a careful treatment of the advection is still of primary importance in the presence of sharp gradients. Indeed, sharp gradients seem as discontinuities on the scale of a mesh spacing.

Our discretization of the advection term is based on the central-difference scheme introduced by Kurganov and Tadmor (KT) [16]. Central schemes tend to be simpler than their upwind counterpart and can more easily be used and adapted as "black box" solvers. The KT scheme was found to introduce less numerical viscosity than earlier central schemes $[19,14]$; its other advantage is that it can be brought to a simple semidiscrete formulation by letting the time step $k$ tend to zero; thus it is possible to consider the spatial discretization independently from the temporal discretization, which can then be implemented by any standard ODE solver.

Conservative schemes are based on an integral representation of the conservation law; the discrete variables represent averages of the conserved quantities - here vorticity - over control cells. Due to conservation, the rate of change of the mean vorticity equals to the integral of the vorticity flux over the cell's boundaries. We take for control cells squares centered at the grid points. Every time step consists of the following steps. (i) Reconstruction of point values from the given cell averages; for a second-order scheme the reconstructed field is piecewise-linear. (ii) Evaluation of the fluxes at the cell's boundaries; because the reconstructed solution might be discontinuous, a careful treatment is necessary. The KT scheme introduces at the cells' interfaces local control volumes of adaptive size over which the discontinuous behavior is integrated; thus, Riemann solvers are avoided. (iii) Update of cell averages by integrating the fluxes using an appropriate quadrature rule.

Specifically, the spatial discretization of $\boldsymbol{\nabla} \cdot(\omega \mathbf{u})$ proceeds as follows.

- Using the standard 5-point Laplacian, we obtain a second-order approximation for the cell-average vorticity,

$$
\omega_{i, j}=\Delta_{h} \psi_{i, j}=\frac{1}{h^{2}}\left(\psi_{i+1, j}+\psi_{i-1, j}+\psi_{i, j+1}+\psi_{i, j-1}-4 \psi_{i, j}\right),
$$

valid in all interior cells, $i, j=1, \ldots, N-1$; our scheme does not require the evaluation of vorticity at boundary cells.

- We proceed with a piecewise-linear reconstruction of the vorticity,

$$
\omega(x, y)=\sum_{i, j}\left[\omega_{i, j}+\left(\omega_{x}\right)_{i, j}\left(x-x_{i}\right)+\left(\omega_{y}\right)_{i, j}\left(y-y_{j}\right)\right] \chi_{i, j}(x, y),
$$

where $\chi_{i, j}$ is the indicator function of the $(i, j)$ cell. The numerical slopes, $\left(\omega_{x}\right)_{i, j}$ and $\left(\omega_{y}\right)_{i, j}$, can be evaluated by simple central differencing when the 
solution is smooth (on the scale of a mesh spacing). Otherwise, nonlinear slope limiters should be used-for example, the min-mod limiters

$$
\left(\omega_{x}\right)_{i, j}=\frac{1}{h} \operatorname{minmod}\left[\theta\left(\omega_{i+1, j}-\omega_{i, j}\right), \frac{1}{2}\left(\omega_{i+1, j}-\omega_{i-1, j}\right), \theta\left(\omega_{i, j}-\omega_{i-1, j}\right)\right]
$$

with $1<\theta<2$. In particular, we evaluate the vorticity at the centers of the cells' edges:

$$
\begin{gathered}
\omega_{i, j}^{E, W}=\omega_{i, j} \pm \frac{1}{2} h\left(\omega_{x}\right)_{i, j}, \\
\omega_{i, j}^{N, S}=\omega_{i, j} \pm \frac{1}{2} h\left(\omega_{y}\right)_{i, j}
\end{gathered}
$$

with the superscripts $W=$ "west," $E=$ "east," $S=$ "south," and $N=$ "north" referring to the orientations of the four edges.

- We then evaluate the normal velocities on the cells' edges by simple secondorder averaging [18]:

$$
\begin{aligned}
& u_{i-\frac{1}{2}, j}=\frac{1}{2}\left(u_{i, j}+u_{i-1, j}\right), \\
& v_{i, j-\frac{1}{2}}=\frac{1}{2}\left(v_{i, j}+v_{i, j-1}\right) .
\end{aligned}
$$

- At each edge we define a numerical flux

$$
\begin{aligned}
& H_{i-\frac{1}{2}, j}=\frac{1}{2}\left(\omega_{i, j}^{W}+\omega_{i-1, j}^{E}\right) u_{i-\frac{1}{2}, j}-\frac{1}{2}\left|a_{i-\frac{1}{2}, j}\right|\left(\omega_{i, j}^{W}-\omega_{i-1, j}^{E}\right), \\
& H_{i, j-\frac{1}{2}}=\frac{1}{2}\left(\omega_{i, j}^{S}+\omega_{i, j-1}^{N}\right) v_{i, j-\frac{1}{2}}-\frac{1}{2}\left|a_{i, j-\frac{1}{2}}\right|\left(\omega_{i, j}^{S}-\omega_{i, j-1}^{N}\right),
\end{aligned}
$$

where the first term on the right-hand side is the average of the one-sided flux evaluations, whereas the second term is a correction that arises from the more precise treatment at the discontinuous boundaries; the prefactors $a_{i-\frac{1}{2}, j}$ and $a_{i, j-\frac{1}{2}}$ correspond to the local characteristic speeds at the cells' interfaces, which in our case are simply the normal velocities, $u$ and $v$, respectively.

- Finally, the divergence of the flux is approximated by

$$
[\boldsymbol{\nabla} \cdot(\omega \mathbf{u})]_{i, j}=\frac{H_{i+\frac{1}{2}, j}-H_{i-\frac{1}{2}, j}}{h}+\frac{H_{i, j+\frac{1}{2}}-H_{i, j-\frac{1}{2}}}{h} .
$$

4.2.2. The viscous term. We next address the spatial discretization, $\Delta_{h}^{2}$, of the biharmonic operator. The standard second-order discretization uses a 13-point stencil. Noncompact stencils are problematic from the point of view of linear solvers. An alternative representation of the discrete biharmonic operator that uses a compact 9-point stencil was developed by Altas et al. [1], and its one-dimensional version was presented in section 3. The idea is to express $\Delta_{h}^{2} \psi_{i, j}$ in terms of the grid values of $\psi$ and its first derivatives, $\psi_{x}=v$ and $\psi_{y}=-u$. A second-order approximation of the biharmonic operator can then be written as

$$
\Delta_{h}^{2} \psi_{i, j}=\frac{12}{h^{2}}\left(-\frac{4}{3} \Delta_{h} \psi_{i, j}+\frac{1}{3} \tilde{\Delta}_{h} \psi_{i, j}+D_{x}^{0} v_{i, j}-D_{y}^{0} u_{i, j}\right),
$$


where $D_{x, y}^{0, \pm}$ are the standard differencing operators and $\tilde{\Delta}_{h}$ is the star-Laplacian

$$
\tilde{\Delta}_{h} \psi_{i, j}=\frac{1}{2 h^{2}}\left(\psi_{i+1, j+1}+\psi_{i-1, j+1}+\psi_{i+1, j-1}+\psi_{i-1, j-1}-4 \psi_{i, j}\right) .
$$

In addition, we need fourth-order expressions for $u_{i, j}$ and $v_{i, j}$ :

$$
\begin{aligned}
& \left(I+\frac{1}{6} h^{2} D_{x}^{+} D_{x}^{-}\right) v_{i, j}=+D_{x}^{0} \psi_{i, j}, \\
& \left(I+\frac{1}{6} h^{2} D_{y}^{+} D_{y}^{-}\right) u_{i, j}=-D_{y}^{0} \psi_{i, j} .
\end{aligned}
$$

These approximations are valid for all interior points; at boundary points $\psi, u$, and $v$ are prescribed by the boundary conditions.

4.3. The linear solver. From a computational point of view, the most timeconsuming part of the computation is the solution of a linear system of the form

$$
\left(\Delta_{h}-\alpha \Delta_{h}^{2}\right) \psi_{i, j}=\operatorname{rhs}_{i, j},
$$

which results from the spatial discretization of (4.1); two such linear systems need to be solved at every time step. Standard iterative methods are known to converge very slowly, if at all, for biharmonic operators.

Biharmonic systems that use the compact stencil representation (4.3), (4.4) can be solved very efficiently with AMG solvers. AMG methods are powerful techniques for the solution of sparse linear systems. They are "black box" solvers, in the sense that they treat the problem to be solved as a pure algebraic system, without reference to the geometrical interpretation of the transition between coarse and fine grids. The advantage of such an approach is that it is readily portable to more complicated systems of coordinates and geometries.

The principle of AMG methods can be summarized as follows. Given an $n$ dimensional linear system $A x=b$, an $m \times n$ restriction matrix $R$ is generated by an algorithm that inspects the graph of the matrix $A$. The $m$-dimensional vector $R x$ is the projection of $x$ on the restricted ("coarse") subspace. The transpose of the restriction matrix, $I=R^{T}$, is used as an interpolation matrix to revert back to the original ("fine") space. AMG methods are based on the presumption that if the righthand side vector, $b$, is sufficiently "smooth" (in a sense that needs to be specified), then the solution $x$ is close to the range of the interpolation matrix $I$, i.e., there exists an $m$-dimensional vector $y$ such that $x \approx I y$. Multiplying the system $A x=b$ by $R$ on the left and approximating $x$ by $I y$, we obtain the restricted system

$$
(R A I) y=R b .
$$

This two-level approach can be applied recursively to form a multilevel method. It then remains to introduce an appropriate "smoother" to operate on the solution before and after being projected to the lower-dimensional subspace.

AMG solvers vary in the way they generate the restriction matrix, in the choice of smoothers, and in the choice of multigrid cycles. Our coarsening method is based on a red-black coloring algorithm developed by Kickinger [13]. Gauss-Seidel iterations have been used for smoothing. Each multigrid cycle starts from the finest level down to the coarsest level and back up (V-cycle). For a $128 \times 128$ grid (that is, a linear 
system of dimension $3 \times 128 \times 128$ ), about ten multigrid cycles with two pre- and postsmoothing steps were needed to reduce the error norm to $10^{-8}$.

There are a number of implementational issues. As long as the time step is not modified, the linear operator is unchanged. In such a case, it is efficient to compute the set of restriction matrices, $R$, with the corresponding linear operators, $R A I$, once, and store them. Most of the computational time is then spent on sparse matrix-vector multiplications, which can be parallelized easily.

5. Numerical results. We have tested our numerical scheme on a classical benchmark problem: flow in a lid-driven cavity. The fluid is confined in a square domain, $\Omega=[0,1]^{2}$, and is driven by the transversal motion of its boundaries. This setup is a challenging test problem, in particular, because the velocity field is discontinuous at the corners adjacent to the moving boundaries, and the viscous stresses diverge logarithmically. (In a method that uses the primitive variables $(\mathbf{u}, p)$, one faces the logarithmic divergence of the pressure.)

We first conducted convergence tests to obtain error estimates and assess the order of accuracy. In Table 1 we show the discrete $L_{2}$ norm $\left\|\psi_{N+1}-\psi_{2 N+1}\right\|_{2}$, where $\psi_{M}$ denotes the computational solution on an $M \times M$ grid. The initial conditions are

$$
\psi(x, y, 0)=\frac{1}{\pi} \sin ^{2}(\pi x) \sin ^{2}(\pi y),
$$

and the boundaries are stationary; the Reynolds number here is $10^{3}$. For short times the convergence rate seems to be less than expected; this is because the errors are very small and therefore dominated by the tolerance specified for the linear solver. For times longer than $t=0.4$ we get an estimated second-order convergence, as expected. Similar results were found for a range of Reynolds numbers between $10^{2}$ and $10^{4}$.

TABLE 1

Error estimate and convergence test for the initial conditions (5.1) and Reynolds number $10^{3}$.

\begin{tabular}{|l||c|c|c|}
\hline Time & $\left\|\psi_{33}-\psi_{65}\right\|_{2}$ & $\left\|\psi_{65}-\psi_{129}\right\|_{2}$ & Rate \\
\hline 0.1 & $4.1 \times 10^{-5}$ & $1.3 \times 10^{-5}$ & 1.64 \\
0.2 & $7.2 \times 10^{-5}$ & $2.1 \times 10^{-5}$ & 1.81 \\
0.3 & $9.5 \times 10^{-5}$ & $2.5 \times 10^{-5}$ & 1.90 \\
0.4 & $1.2 \times 10^{-4}$ & $2.9 \times 10^{-5}$ & 1.99 \\
0.5 & $1.4 \times 10^{-4}$ & $3.3 \times 10^{-5}$ & 2.08 \\
\hline
\end{tabular}

We next display results for lid-driven flows. For low enough Reynolds numbers the flow approaches a steady state; the lower the Reynolds number is, the shorter the transient is. In Figure 1(a) we display stream function contour lines in the steady state for $\operatorname{Re}=400$. The fluid is initially at rest, and it is driven impulsively by the rightward motion of the top boundary. For comparison we display the same level sets as in [9, Figure 3 and Table III]. In Figure 1(b), (c) we plot the steady-state profile of the $u(v)$ component of the velocity as a function of $y(x)$ at $x=0.5(y=0.5)$. The solid line represents our results, whereas the symbols are data reported in [9]). The agreement is excellent. In Table 2 we list the minimum value of the stream function, which takes place in the core of the primary vortex, at different times and for three different grid sizes. For a grid size of $128 \times 128$ the results seem to have fully converged. It takes about 35 time units to reach a steady flow; the minimum value of the stream function is then -0.1140 ; in [9] the reported value is -0.1139 ; the same value has also been reported by Pan and Glowinski for a slightly regularized flow [20]. We also compare extremal values of the stream function for the secondary 

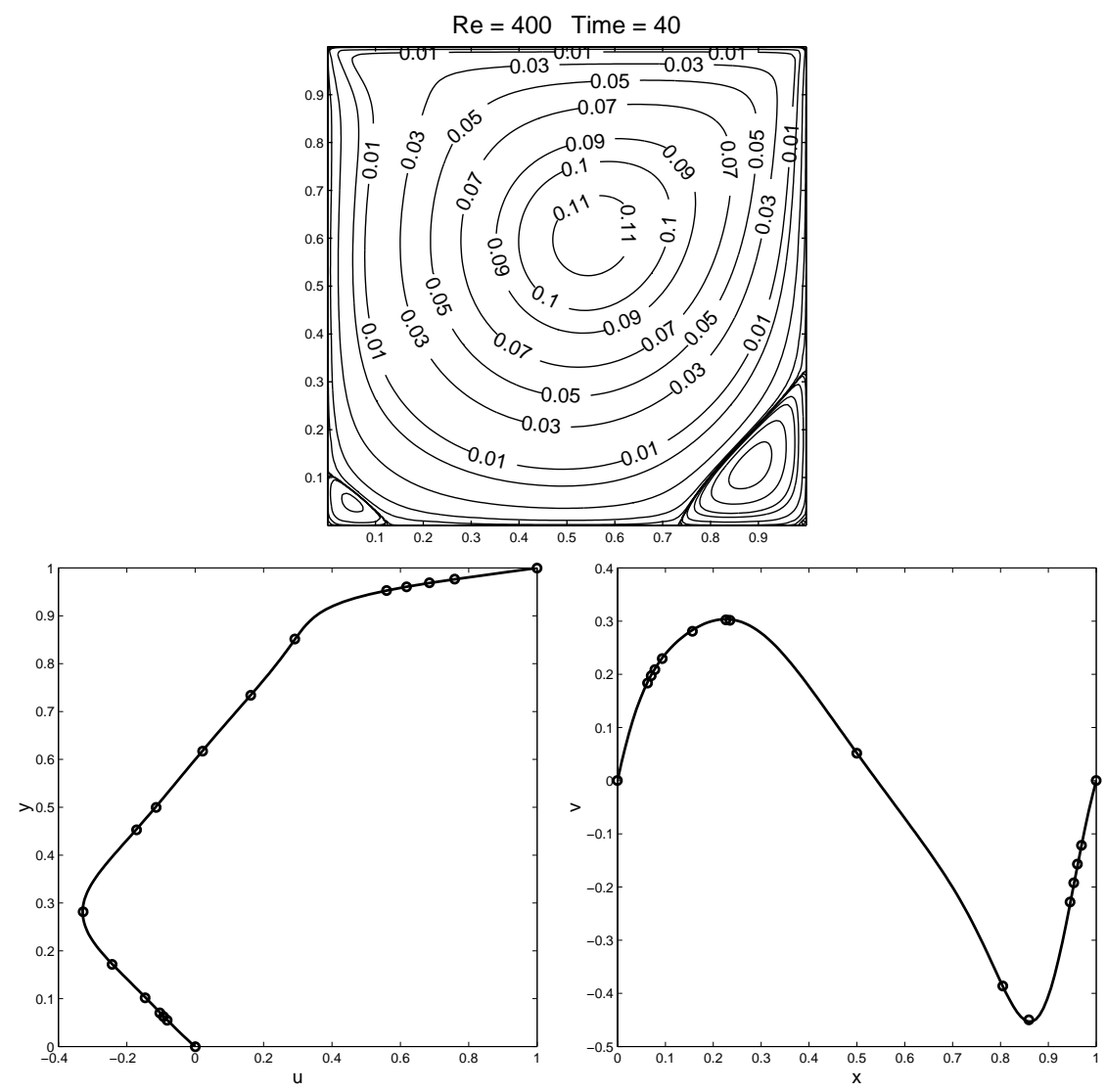

FIG. 1. (a) Contour plots of the stream function at time $t=40$ after an impulsive start at Reynolds number Re $=400$. The top boundary moves to the right with velocity $u=1$. (b) Steadystate profile of the $u$ velocity component as a function of $y$ at $x=0.5$. (c) Steady-state profile of the $v$ velocity component as a function of $x$ at $y=0.5$.

TABLE 2

Minimum value of the stream function at different times and for different mesh sizes. The Reynolds number is $R e=400$.

\begin{tabular}{|l||c|c|c|}
\hline Time & $64 \times 64$ & $96 \times 96$ & $128 \times 128$ \\
\hline$t=5.0$ & -0.09062 & -0.09074 & -0.09076 \\
$t=15.0$ & -0.11164 & -0.11173 & -0.11174 \\
$t=25.0$ & -0.11378 & -0.11385 & -0.11385 \\
$t=35.0$ & -0.11393 & -0.11400 & -0.11401 \\
\hline
\end{tabular}

vortices. For the bottom-right secondary vortex the maximum value of the stream function is $6.579 \times 10^{-4}$, and it is $1.404 \times 10^{-5}$ for the bottom-left vortex; the numbers reported in [9] are $6.423 \times 10^{-4}$ and $1.419 \times 10^{-5}$, respectively. In [11] the time to reach a steady state was estimated to be about 46 time units. A precise quantitative comparison is hard to perform due to the arbitrary nature of the stopping criterion.

Similar calculations were carried out for $\mathrm{Re}=5000$. In Figure 2(a) we plot the minimum value of the stream function versus time. Steady-state velocity profiles are shown in Figure 2(b), (c). Snapshots of stream function contour lines are presented in Figure 3. Note the much longer transient; its takes about 340 time units to reach 

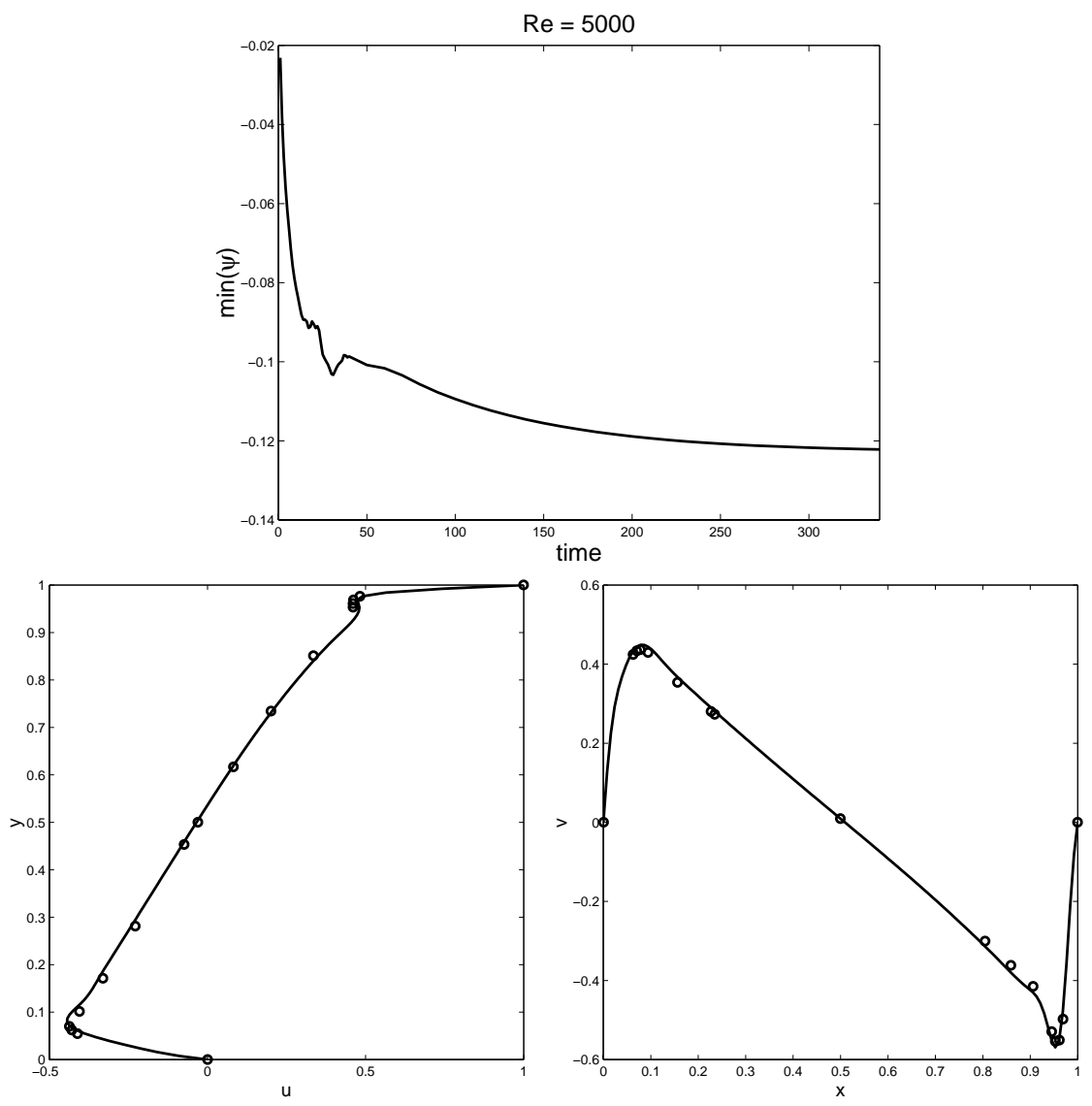

FIG. 2. (a) Minimum value of the stream function versus time for Re $=5000$. (b) Steady-state profile of the $u$ velocity component as a function of $y$ at $x=0.5$. (c) Steady-state profile of the $v$ velocity component as a function of $x$ at $y=0.5$.

a steady flow. Note also the nonmonotonic behavior of the minimum value of the stream function, which reflects the fact that recirculation zones are created and annihilated along the side and bottom walls until the final vorticity pattern emerges. Such dynamics were also reported in [11] and are consistent with subcritical behavior prior to an oscillatory instability, whose occurrence has been predicted in [21, 20]. Eventually, the stream function reaches the value of -0.122160 ; in [9] the predicted value is -0.118966 , whereas in [20] it is -0.121218 . The velocity profiles are again in excellent agreement with the data reported in [9].

In a recent paper, Pan and Glowinski [20] obtained limit cycle solutions for $\mathrm{Re}=$ 8500. The occurrence of a Hopf bifurcation has been speculated before [21] but was believed to take place at a significantly higher Reynolds number. Our results for $\operatorname{Re}=8500$ support the findings of [20]. In Figure 4 we plot the time evolution of the kinetic energy over a time interval of $t=3$; the function is oscillatory with a period of about 2.5; the period reported in [20] is 2.27. In Figure 5 we show a complete cycle of stream function contours during a time interval of 2.5. The primary vortex remains practically unchanged, variations being noticeable only within the secondary vortices. It takes about 200 time units to reach this state starting from a fluid at rest. 

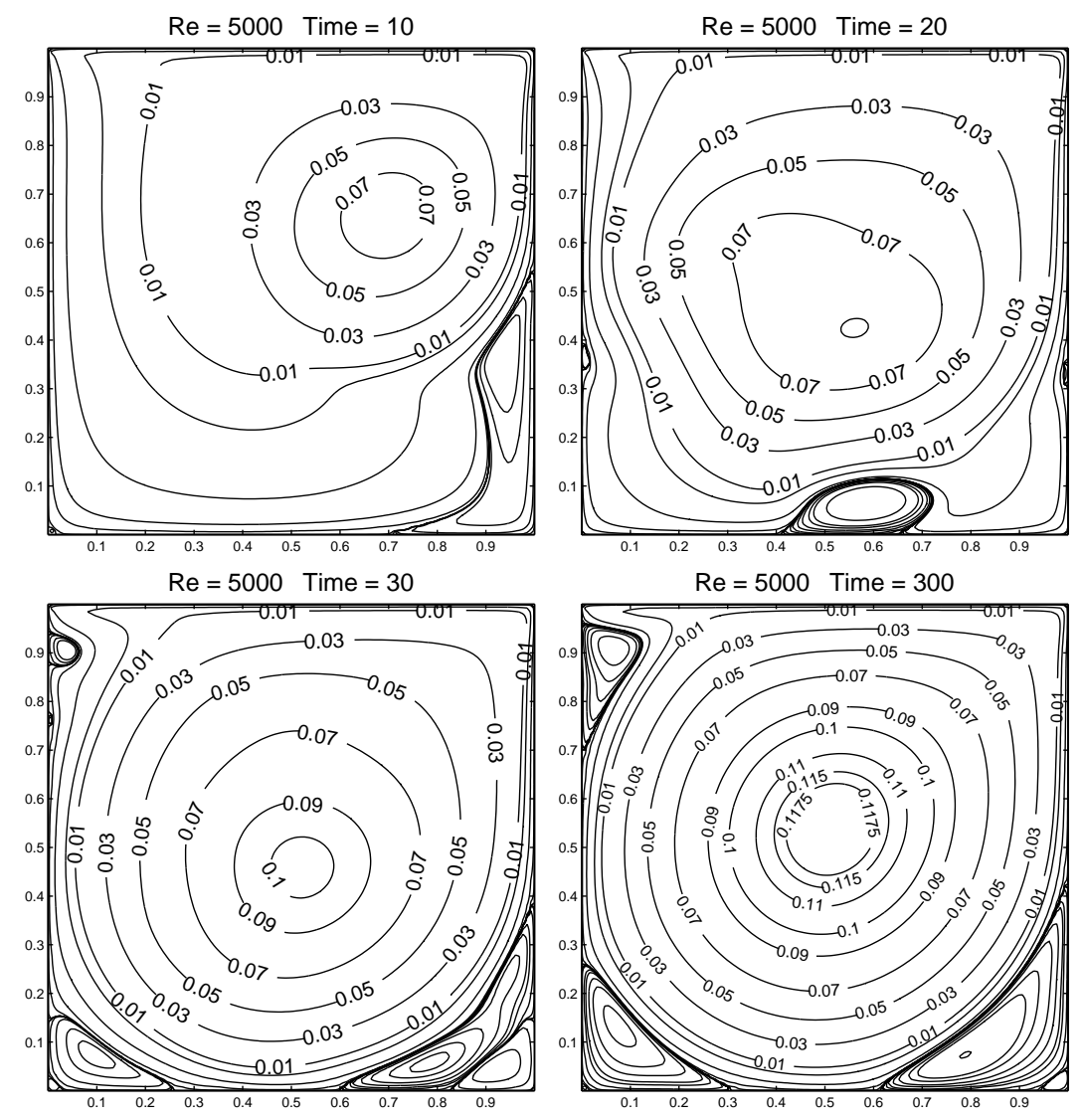

FIG. 3. Contour plots of the stream function. The top boundary moves to the right with velocity $u=1$. The Reynolds number is Re $=5000$. The contours are shown for time $t=10, t=20, t=30$, and $t=300$. The grid size is $128 \times 128$.

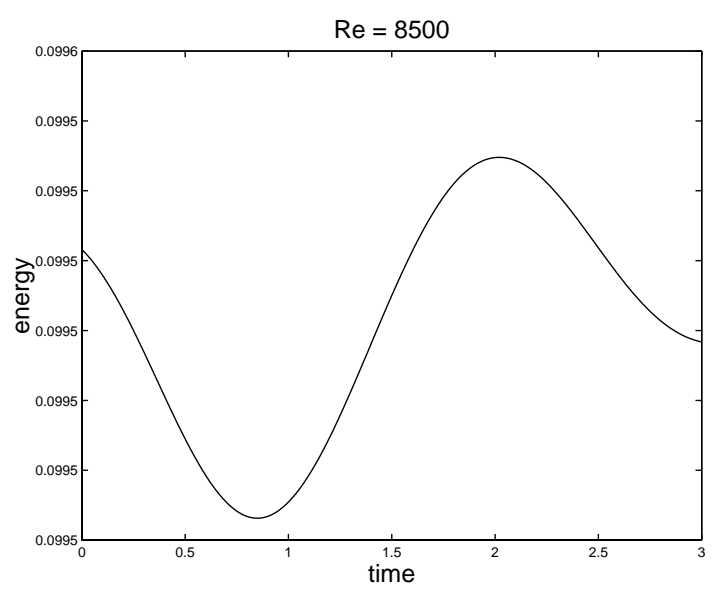

FIG. 4. Kinetic energy versus time for $R e=8500$. 

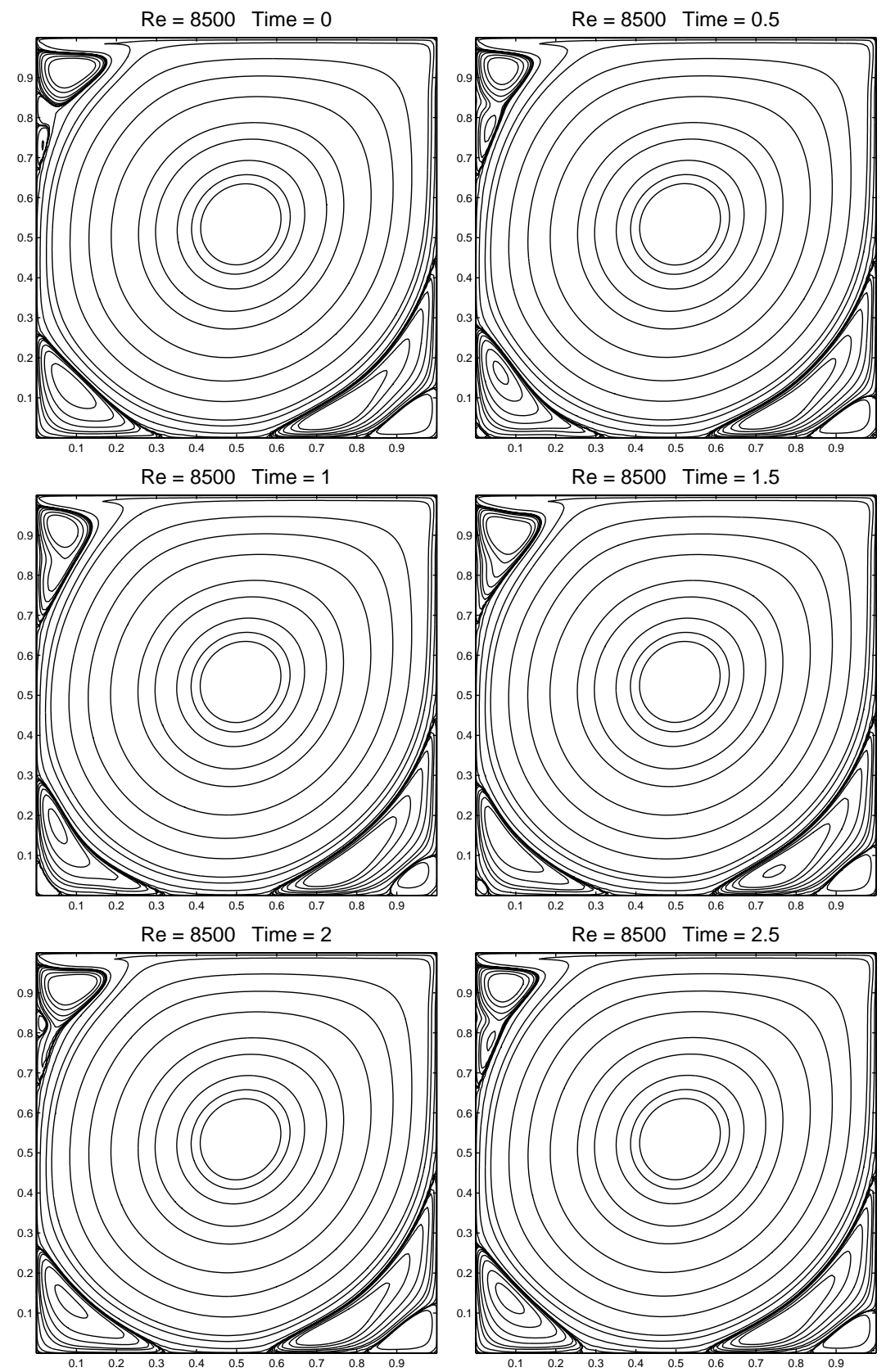

FIG. 5. Contour plots of the stream function. The top boundary moves to the right with velocity $u=1$. The Reynolds number is Re $=8500$. This sequence covers one period of the limit cycle. The grid size is $128 \times 128$.

For even higher Reynolds numbers the flows are much more complex. An Re $=$ 20000 flow is shown in Figure 6, where we display snapshots of vorticity contour lines for a fluid that is driven by the upward motion of its left and right boundaries. Narrow and concentrated shear layers are generated along the moving boundaries and 

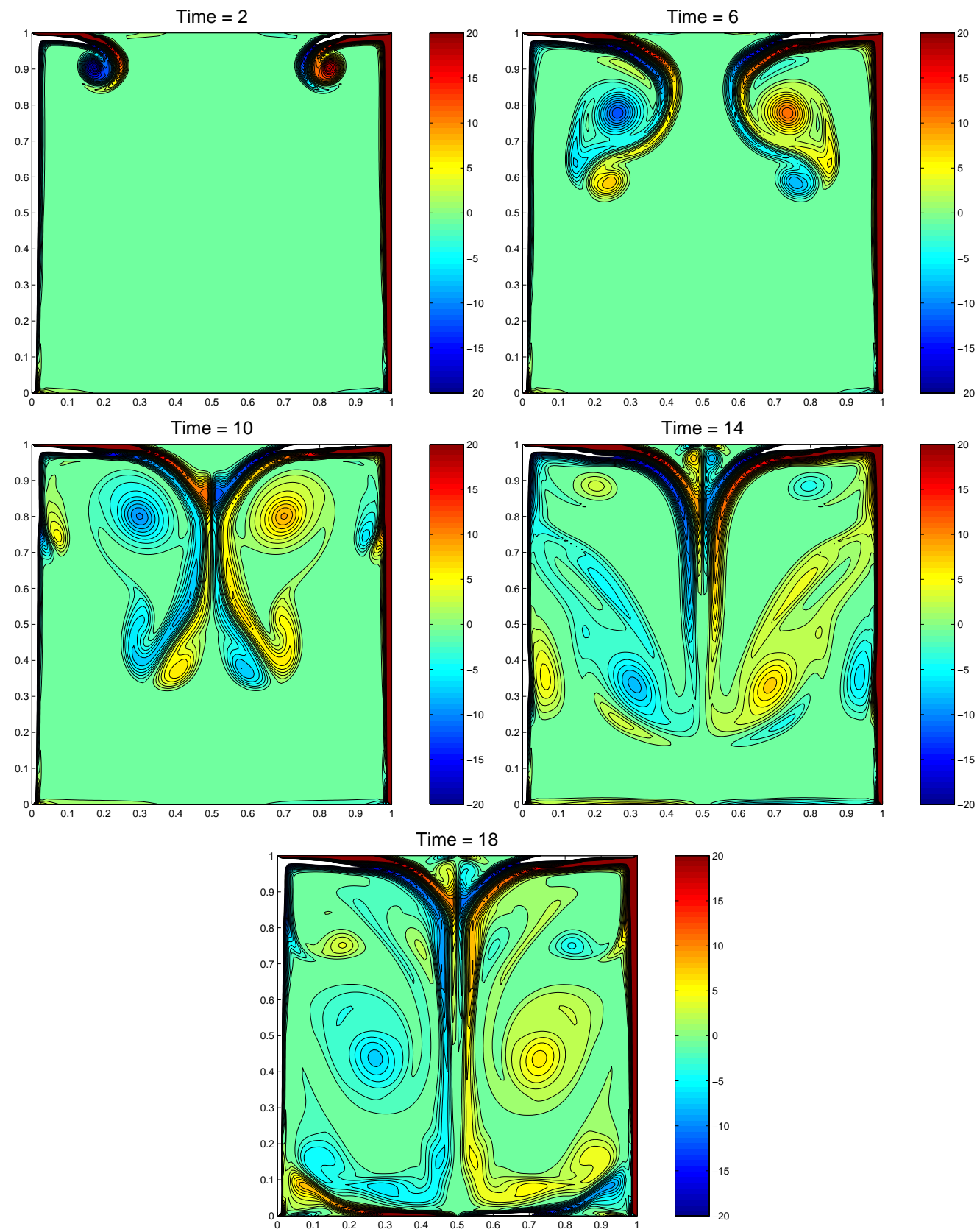

FIG. 6. Contour plots of vorticity. The left and right walls move upward with velocity $v=1$. The Reynolds number is Re $=20000$. The contours are shown for time $t=2, t=6, t=10, t=14$, and $t=18$. The grid size is $128 \times 128$.

transported with the flow. The shear layers are unstable and rapidly intermingle to form a convoluted vorticity pattern. This sequence of vorticity contours demonstrates the robustness of the scheme. The vorticity gradients are large with sharp variations over single cells. 
6. Concluding remarks. The present scheme is based on the paradigm that has been established in [4], whereby vorticity dynamics should be viewed as a projection of stream function dynamics; thus vorticity boundary conditions are totally avoided, and natural boundary conditions are imposed on the stream function. Numerical methods based on stream function variables are by themselves not a novel idea. What have been missing for many years are accurate and efficient ways of implementation. We make no claim, however, that methods based on vorticity boundary conditions are invalid. Such methods have been proven to work within the frameworks of both difference schemes and vortex methods.

An important property of the proposed scheme is its modularity. It is not restricted to a specific type of hyperbolic or biharmonic solver, and each of its elements can be implemented in various ways. In particular, higher-order spatio-temporal discretizations are relatively easy to implement (see, e.g., [1] for a fourth-order discretization of the biharmonic equation and [15] for a third-order version of the KT scheme). Finally, an extension to three dimensions seems realizable.

Acknowledgments. I am grateful to Professors M. Ben-Artzi, D. Fishelov, and S. Trachtenberg for introducing me to stream function dynamics and for making their work and data accessible. This paper has been motivated and influenced by their work. I have benefited from useful discussions with Professor A. Chorin, Mr. Y. Farjoun, Professor R. Glowinski, Professor O. Hald, Professor E. Tadmor, and Dr. D. von Terzi. Special thanks to Mr. Y. Farjoun, who implemented the multigrid solver.

\section{REFERENCES}

[1] I. Altas, J. Dym, M. M. Gupta, and R. P. Manohar, Multigrid solution of automatically generated high-order discretizations for the biharmonic equation, SIAM J. Sci. Comput., 19 (1998), pp. 1575-1585.

[2] C. Anderson AND M. Reider, A high order explicit method for the computation of flow about a circular cylinder, J. Comput. Phys., 125 (1996), pp. 207-224.

[3] F. Auteri and L. Quartapelle, Galerkin spectral method for the vorticity and stream function equations, J. Comput. Phys., 149 (1999), pp. 306-332.

[4] M. Ben-Artzi, D. Fishelov, and S. Trachtenberg, Vorticity dynamics and numerical resolution of the incompressible Navier-Stokes equations, Math. Model. Numer. Anal., 35 (2001), pp. 313-330.

[5] A. Chorin, On the convergence of discrete approximations to the Navier-Stokes equations, Math. Comp., 22 (1969), pp. 745-762.

[6] A. Chorin, Numerical study of slightly viscous flow, J. Fluid Mech., 57 (1973), pp. 785-796.

[7] W. E AND J.-G. LiU, Essentially compact schemes for unsteady viscous incompressible flows, J. Comput. Phys., 126 (1996), pp. 122-138.

[8] W. E AND J.-G. LiU, Vorticity boundary condition and related issues for finite difference schemes, J. Comput. Phys., 124 (1996), pp. 368-382.

[9] U. Ghia, K. GhiA, And C. Shin, High-Re solutions for incompressible flow using the NavierStokes equations and a multigrid method, J. Comput. Phys., 48 (1982), pp. 387-411.

[10] E. Godlewski and P.-A. Raviart, Hyperbolic Systems of Conservation Laws, Math. Appl. 3-4, Ellipses, Paris, 1991.

[11] J. GoOdRICH AND W. SoH, Time-dependent viscous incompressible Navier-Stokes equations: The finite difference Galerkin formulation and stream function algorithms, J. Comput. Phys., 84 (1989), pp. 207-241.

[12] B. Gustafsson, H.-O. Kreiss, and J. Oliger, Time Dependent Problems and Difference Methods, John Wiley and Sons, New York, 1995.

[13] F. Kickinger, Algebraic multigrid for discrete elliptic second-order problems, in Multigrid Methods, Proceedings of the Fifth European Multigrid Conference, Stuttgart, Germany, Springer-Verlag, Berlin, 1996, pp. 157-172.

[14] R. Kupferman and E. TAdmor, A fast high-resolution second-order central scheme for incompressible flows, Proc. Natl. Acad. Sci. USA, 94 (1997), pp. 4848-4852. 
[15] A. Kurganov And D. Levy, Third-order semidiscrete central scheme for conservation laws and convection-diffusion equations, SIAM J. Sci. Comput., 22 (2000), pp. 1461-1488.

[16] A. Kurganov and E. TAdmor, New high-resolution central schemes for nonlinear conservation laws and convection-diffusion equations, J. Comput. Phys., 160 (2000), pp. 214-282.

[17] R. LeVeque, Numerical Methods for Conservation Laws, Birkhauser Verlag, Basel, 1992.

[18] D. LeVy AND E. TADMOR, Non-oscillatory central schemes for the incompressible 2-D Euler equations, Math. Res. Lett., 4 (1997), pp. 1-20.

[19] H. Nessyahu and E. TAdmor, Non-oscillatory central differencing for hyperbolic conservation laws, J. Comput. Phys., 87 (1990), pp. 408-463.

[20] T. PAN AND R. GLOwinski, A projection/wave-like equation method for the numerical simulation of incompressible viscous fluid flow modeled by the Navier-Stokes equations, Comput. Fluid Dyn. J., 9 (2000), pp. 28-42.

[21] J. Shen, Hopf bifurcation of the unsteady regularized driven cavity flow, J. Comput. Phys., 95 (1991), pp. 228-245.

[22] A. Тном, The flow past circular cylinders at low speeds, Proc. Roy. Soc. London Sect. A, 141 (1933), p. 651. 